\title{
PREVALENCIA Y CARACTERIZACIÓN DE LAS DISFUNCIONES SEXUALES EN MUJERES, EN 12 CIUDADES COLOMBIANAS, 2009-2016
}

\section{Prevalence and characterisation of sexual dysfunctions in women, in 12 Colombian cities, 2009-2016}

Franklin José Espitia-De La Hoz, MD

Recibido: julio 4/17 - Aceptado: marzo 7/18

\section{RESUMEN}

Objetivo: establecer la prevalencia y caracterizar la disfunción sexual en una población de mujeres sexualmente activas, con edades entre 18 y 72 años, en doce ciudades colombianas.

Materiales y métodos: estudio de corte transversal descriptivo. Se incluyeron mujeres mayores de 18 años con actividad sexual en las últimas 6 semanas, y residentes en Colombia. Se excluyeron las mujeres analfabetas, embarazadas o en primeros 6 meses posparto, pacientes psiquiátricas, con deficit neurológico o historial de cáncer. El estudio se llevó a cabo en la consulta externa ginecológica de instituciones hospitalarias privadas en 12 ciudades del país (Bogotá, Medellín, Cali, Barranquilla, Cartagena, Cúcuta, Ibagué, Bucaramanga, Villavicencio, Pereira, Manizales y Armenia) entre junio de 2009 y diciembre de 2016. Se realizó muestreo consecutivo. Se aplicó el Índice de Función Sexual Femenina validado en español. Se midieron variables sociodemográficas, antecedentes de salud sexual y reproductiva, comportamiento

1 Ginecoobstetra, Universidad Militar Nueva Granada; Uroginecología / FUCS - Hospital de San José / Unicamp, Brasil; MSc en Sexología: Educación y asesoramiento sexual, Universidad de Alcalá de Henares. espitiafranklin@hotmail.com sexual, frecuencia de disfunción sexual global y por tipo de disfunción evaluada. Se realizó un análisis descriptivo de la información utilizando medidas de frecuencia absoluta y relativa para los datos. Se hace descripción estratificada por edad (40 años o menos y mayor de 40 años).

Resultados: de un total de 72.894 mujeres candidatas a ingresar se analizaron finalmente 50.991 (69,95\%). La edad promedio fue de 30,9 $\pm 10,8$ años. La prevalencia de disfunción sexual en el grupo estudiado fue del 32,97\% (16.812 mujeres). La puntuación del Índice de Función Sexual Femenina (IFSF) en las mujeres afectadas fue de $24,07 \pm 6,18$ puntos. Se presentaron dificultades con el deseo (32,97\%), el orgasmo (21,93\%), la excitación (16,86\%), la lubricación (14,79\%) y dolor (7,56\%). La mediana de disfunciones sexuales por mujer fue de 2, que se hizo presente en el 64,16\%. Conclusión: en las mujeres colombianas existe una prevalencia de disfunciones sexuales cercana a la tercera parte de la población, caracterizadas principalmente por trastornos del deseo y del orgasmo. Se requieren intervenciones para establecer un plan diagnóstico y terapéutico inmediato.

Palabras clave: disfunciones sexuales fisiológicas, dispareunia, mujeres, orgasmo, prevalencia. 


\section{ABSTRACT}

Objective: To determine the prevalence and to characterise sexual dysfunction in a population of sexually active women with ages ranging between 18 and 72 years, in 12 Colombian cities.

Materials and methods: Descriptive cross-sectional study that included women 18 years of age and older, sexually active within the past six weeks, living in Colombia. Excluded were illiterate and pregnant women, women in the first 6 months postpartum, women with a psychiatric disease or neurological deficit, and women with a history of cancer. The study was conducted in outpatient gynaecological services of private hospitals in twelve cities (Bogotá, Medellín, Cali, Barranquilla, Cartagena, Cúcuta, Ibagué, Bucaramanga, Villavicencio, Pereira, Manizales and Armenia) between June 2009 and December 2016. A consecutive sampling method was used. The "Female Sexual Function Index" validated in Spanish was applied. Sociodemographic variables, a history of sexual and reproductive health, sexual behaviour, and frequency of overall sexual dysfunction and by type of dysfunction assessed were measured. A descriptive analysis of the data was performed using absolute and relative measurements. A stratified description was made by age under or over 40 years.

Results: Of a total of 72,894 candidates for enrolment, 50,991 (69,95\%) were ultimate analysed. Mean age was $30.9 \pm 10.8$ years. The prevalence of sexual dysfunction in the study group was $32.97 \%$ (16,812 women). The score on the FSFI in the affected women was $24.07 \pm 6.18$ points. Issues were found with libido in $32.97 \%$, orgasm in $21.93 \%$, arousal in $16,86 \%$, lubrication in $14,79 \%$, and pain in $7.56 \%$. Median sexual dysfunction per woman was 2, found in $64.16 \%$.

Conclusion: Among Colombian women, a prevalence of sexual dysfunction is found in close to one-third of the population, characterised mainly by issues with libido and orgasm. Interventions are required in order to establish an immediate diagnostic and therapeutic plan.
Key words: Physiological sexual dysfunctions, dyspareunia, women, orgasm, prevalence.

\section{INTRODUCCIÓN}

La salud sexual ha sido definida como: "la experiencia del proceso continuo de bienestar físico, psicológico y sociocultural relacionado con la sexualidad, respetando el derecho al placer sexual, a la expresión sexual emocional, a la información basada en el conocimiento científico, a la educación sexual integral y a la atención de la salud sexual, los mismos que deben ser reconocidos y garantizados" (1). Una disfunción sexual se define como la dificultad, durante cualquier etapa de la actividad sexual normal, experimentada por un individuo o pareja, durante un mínimo de 6 meses (2), trastorno que podría afectar negativamente su sexualidad y calidad de vida.

Las disfunciones sexuales abarcan cuatro tipos de dificultades o trastornos sexuales: del orgasmo, de la excitación sexual, del deseo sexual y dolor (3). En la mujer, los problemas sexuales se asocian con dificultades maritales, ansiedad y depresión (4), los cuales tienen una repercusión negativa en su calidad de vida, ya que la actividad sexual es importante para la salud general y el bienestar de un individuo (5), así como para la armonía en pareja.

La prevalencia de disfunción sexual varía de manera amplia en la literatura internacional. En Europa se ha reportado entre el $28 \%$ (6) y $53 \%$ (7). En Asia se ha descrito una prevalencia variable entre el 46\% (8) y el $73 \%$ (9). En América se ha informado que fluctúa entre el $43 \%$ (10) y el $65 \%$ (11). Por otra parte, se estima que alrededor del $40 \%$ de las mujeres experimentará algún tipo de trastorno sexual a lo largo de su vida $(12,13)$. La frecuencia de disfunción sexual varía según la edad, la presencia de condiciones mórbidas, antecedentes de violencia sexual, problemas interpersonales, etc.; por lo que se considera un fenómeno multifactorial. Entre los factores de riesgo asociados de forma significativa con el trastorno sobresalen: baja frecuencia de relaciones sexuales, bajo nivel educativo, desempleo, edad mayor 
a 40 años mala comunicación con la pareja, matrimonio de 10 o más años, menopausia, presencia de enfermedad médica de base, presentar una pareja con disfunción sexual y ser ama de casa (14-17).

Se han desarrollado múltiples cuestionarios para el estudio y la evaluación de la función sexual en la mujer, entre ellos están el Brief Index of Sexual Function for Women (BISF-W) (18), el Sexual Function Questionnaire (SFQ) (19), y el Índice de Función Sexual Femenina (IFSF) (en inglés Female Sexual Function Index - FSFI) (20). Este último ha sido el más utilizado en las investigaciones de todo el mundo, además de haberse traducido al español y validado en diferentes países, entre ellos Colombia (20-22). Su principal valor radica en haber sido diseñado, de forma específica, para evaluar la salud sexual femenina y detectar las disfunciones sexuales de la mujer. El IFSF es utilizado, no solo por la alta confiabilidad, sino por propiedades psicométricas y su excelente desempeño (consistencia interna, fiabilidad test-retest y validez discriminante), por lo cual se ha convertido en la herramienta predilecta para evaluar la función sexual femenina, además por ser autoadministrado, breve, rápido, sencillo y confiable a la hora de aplicarlo en un amplio rango de edad, y y de cumplir la clasificación del International Consensus Development Conference on Female Sexual Dysfunctions (14, 20, 22, 23).

Hay limitaciones para evaluar de manera válida y precisa la prevalencia de disfunciones sexuales femeninas debido a que se han utilizado diferentes definiciones de función sexual "normal" y "anormal”, y por la selección de muestras obtenidas de diferente base poblacional (16, 17, 22, 24). En Colombia, las publicaciones acerca de la prevalencia de las disfunciones sexuales de las mujeres tienen limitaciones en cuanto a la cobertura y el tipo de población incluida $(22,23)$; es por eso que el objetivo de este estudio es estimar la prevalencia de las disfunciones sexuales y caracterizar el dominio afectado (deseo, excitación, lubricación, orgasmo, satisfacción y dolor coital) en una muestra amplia de mujeres colombianas.

\section{MATERIALES Y MÉTODOS}

Diseño y población. Estudio de corte transversal descriptivo, en el que se incluyeron mujeres de 18 años o más que manifestaron haber tenido actividad sexual en las últimas seis semanas y firmaron el consentimiento informado para participar en el estudio. Se excluyeron mujeres gestantes o en los primeros 6 meses posparto, mujeres con bajo nivel de escolaridad, con enfermedades psiquiátricas o déficit neurológico, retardo mental e historial de cáncer. Las mujeres fueron vistas en consulta externa de ginecología, de 12 instituciones privadas de alta complejidad en el periodo del 01 de junio de 2009 al 31 de diciembre de 2016. Estas instituciones atienden población perteneciente al régimen contributivo y subsidiado en el sistema de seguridad social en Colombia. Se seleccionaron instituciones en las ciudades de: Bogotá, Medellín, Cali, Barranquilla, Cartagena, Cúcuta, Ibagué, Bucaramanga, Villavicencio, Pereira, Manizales y Armenia. Se realizó muestreo consecutivo de mujeres escogidas por conveniencia. Se intentó incluir el total de la muestra de mujeres que contestaron la encuesta.

Procedimiento. En las instituciones participantes, las mujeres que acudieron a la consulta externa de ginecología fueron evaluadas por una enfermera profesional, a fin de determinar si cumplían con los criterios de elegibilidad del estudio. Si la mujer cumplía con los criterios, se le informaban los objetivos de la investigación, así como el propósito que tenían los resultados, garantizándoseles la confidencialidad de la información, y se le solicitaba la firma del consentimiento informado. Una vez se obtenía el consentimiento las auxiliares de enfermería, encargadas de la recolección del instrumento estandarizado, le daban instrucciones para el autodiligenciamiento del cuestionario IFSF a cada una de las mujeres, de manera individual y en un ambiente privado.

El Índice de Función Sexual Femenina (IFSF) es un instrumento conformado por 19 preguntas. Evalúa 6 dominios: deseo (ítems 1 y 2), excitación (ítems 3 a 6), lubricación (ítems 7 a 10), orgasmo (ítems 11 
a 13), satisfacción (ítems 14 a 16) y dolor durante la relación sexual (ítems 17 a 19). La respuesta se mide según el siguiente puntaje: 0) Sin actividad sexual; 1) Casi nunca; 2) Menos de la mitad de las veces; 3) La mitad de las veces; 4) Más de la mitad de las veces; 5) Casi siempre. La puntuación de cada dominio se multiplica por un factor que oscila entre 0,3 a 0,6 , considerando el dominio evaluado; al final el resultado es la suma aritmética de los dominios; cuanto mayor es el puntaje, mejor es la sexualidad. El rango total de la puntuación del IFSF va desde 2 hasta 36 (Anexo 1); un puntaje inferior o igual a 26,55 puntos, o cuando la puntuación de algún dominio es menor a 3,6 puntos, se considera como criterio de riesgo de disfunción sexual $(12,13,16,22)$.

El equipo de investigación estuvo conformado por el investigador principal quien lideró los equipos de cada ciudad. En cada institución el equipo estaba integrado por tres auxiliares de enfermería entrenadas, las cuales a su vez eran coordinadas por una enfermera profesional, diplomada en sexología clínica; todas eran expertas en el diligenciamiento del IFSF y conocedoras, a cabalidad, de los propósitos de la investigación.

Variables medidas. Sociodemográficas (edad, raza, escolaridad, estrato socioeconómico, estado civil, ocupación, afiliación al sistema general de seguridad social en salud, convivencia, condición espiritual o religiosa, área de residencia); variables de salud sexual y reproductiva (paridad, edad de la menopausia, tabaquismo, ingesta de alcohol, uso de anticoncepción hormonal, antecedente personal y familiar de depresión o de disfunción sexual, uso de terapia hormonal de reemplazo); variables de comportamiento sexual (orientación sexual, edad de la primera relación sexual, recurrencia a la masturbación, coito -vaginal o anal-, frecuencia promedio de relaciones sexuales semanales, frecuencia para lograr alcanzar el orgasmo, número de parejas sexuales, tiempo de convivencia en pareja, antecedente de abuso sexual o violencia sexual en el matrimonio, pareja con disfunción sexual e infidelidad). Se indagaron además las preguntas de los dominios de la encuesta IFSF; adicionalmente, se consideró el análisis por subgrupos de edad (menores y mayores de 40 años) para hacer una comparación final del comportamiento porcentual de la prevalencia de disfunciones sexuales en las mujeres en estos dos periodos.

Análisis estadístico. Los cálculos estadísticos se realizaron con el programa EPIDAT 3.1. Las variables cualitativas fueron expresadas como frecuencias absolutas y relativas (porcentajes), y las cuantitativas como media y desviación estándar (DE). Los resultados se exponen de manera agrupada para el total de la población. Se presenta la prevalencia de disfunción sexual global y por dominios.

Aspectos éticos. La investigación fue aprobada por el Comité Ético Científico del Servicio de Salud correspondiente en cada institución; se solicitó la firma del consentimiento informado antes de ingresar a la investigación, se garantizó la confidencialidad de la información de las mujeres participantes.

\section{RESULTADOS}

Un total de 72.894 mujeres fueron invitadas a participar en el estudio; de estas, 3.801 (5,21\%) no aceptaron participar. A las 69.093 mujeres restantes se les solicitó diligenciar el IFSF, pero un total de $12.084(16,57 \%)$ se retiraron voluntariamente por sentirse incómodas al responder ciertas variables relacionadas con su salud sexual, quedando 57.009 encuestas, de las cuales se encontraron 6.018 $(8,25 \%)$ formularios con fallas en el diligenciamiento, los que se excluyeron. De esta manera, para el análisis final se tomó en cuenta un total de 50.991 (69,95\%) formularios (figura 1).

Las características sociodemográficas de la población de las mujeres entrevistadas mostraron que la edad promedio fue de 30,9 (DE $\pm 10,8)$ años, la mayoría eran mestizas (59,3\%). El 76,94\% estaban casadas o en unión libre. El 60,3\% profesaban la religión católica. Se encontró educación secundaria en el 54,04\%, y el 35,09\% contaba con título universitario. Respecto al estrato socioeconómico de la población se observó que el 16,14\% eran de estrato 
alto. El 52,63\% eran amas de casa, el 72,28\% pertenecían al régimen contributivo de seguridad social en salud, y el 79,17\% residían el área urbana. La edad de la menopausia fue de 49,8 \pm 3,6 años. La relación multípara a nulípara fue de 3:1.

Los antecedentes de salud sexual y reproductiva mostraron una mediana de 3 hijos en la paridad (2 vía vaginal y 1 vía cesárea por mujer), y un rango entre 0 y 9 hijos. Un total de 40.727 (79,87\%) mujeres informaron haber tenido más de un embarazo, y de estos 32.517 (79,84\%) fueron no planeados. El 12,6\% eran fumadoras habituales, mientras que $14,7 \%$ se declararon como exfumadoras. El $66,3 \%$ consumían alcohol. Una alta proporción de las mujeres utilizaban métodos anticonceptivos $(89,83 \%)$ con predominio de la anticoncepción hormonal (58,99\%), con la píldora en primer lugar (78,75\%), seguida del implante $(17,98 \%)$ y, por último, la inyección $(3,25 \%)$, mientras que el uso de terapia hormonal de reemplazo solo se encontró en el 5,99\% de las mujeres mayores de 40 años. Por otra parte, 3.564 mujeres (6,98\%) manifestaron que el embarazo había sido producto de una relación sexual bajo efectos del alcohol y sin preservativo. La prevalencia global de abortos fue del 17,21\%, de los cuales en 3.456 mujeres (7,26\%) fueron inducidos y realizados en condiciones de riesgo (uso de medicamentos autoformulados o en sitios clandestinos).

En cuanto a los comportamientos sexuales, el número de parejas sexuales reportó una mediana de 12, con un rango entre 1 y 18. El 26,98\% refirió más de 10 años de tiempo de convivencia en pareja. El 68,17\% manifestó que la pareja presentaba alguna disfunción sexual. El 34,28 \% refirió que la pareja le había sido infiel. El 19,3\% afirmó haber sido infiel; de estas, el 26,99\% refirió haber sido infiel en una ocasión, 52,68 \% más de una y menos de cincos veces, 6,91\% refirió serlo más de cinco veces, y el 13,4\% refirió ser infiel con frecuencia. Las características generales de las pacientes se

Figura 1.

Diagrama de flujo de la población del estudio

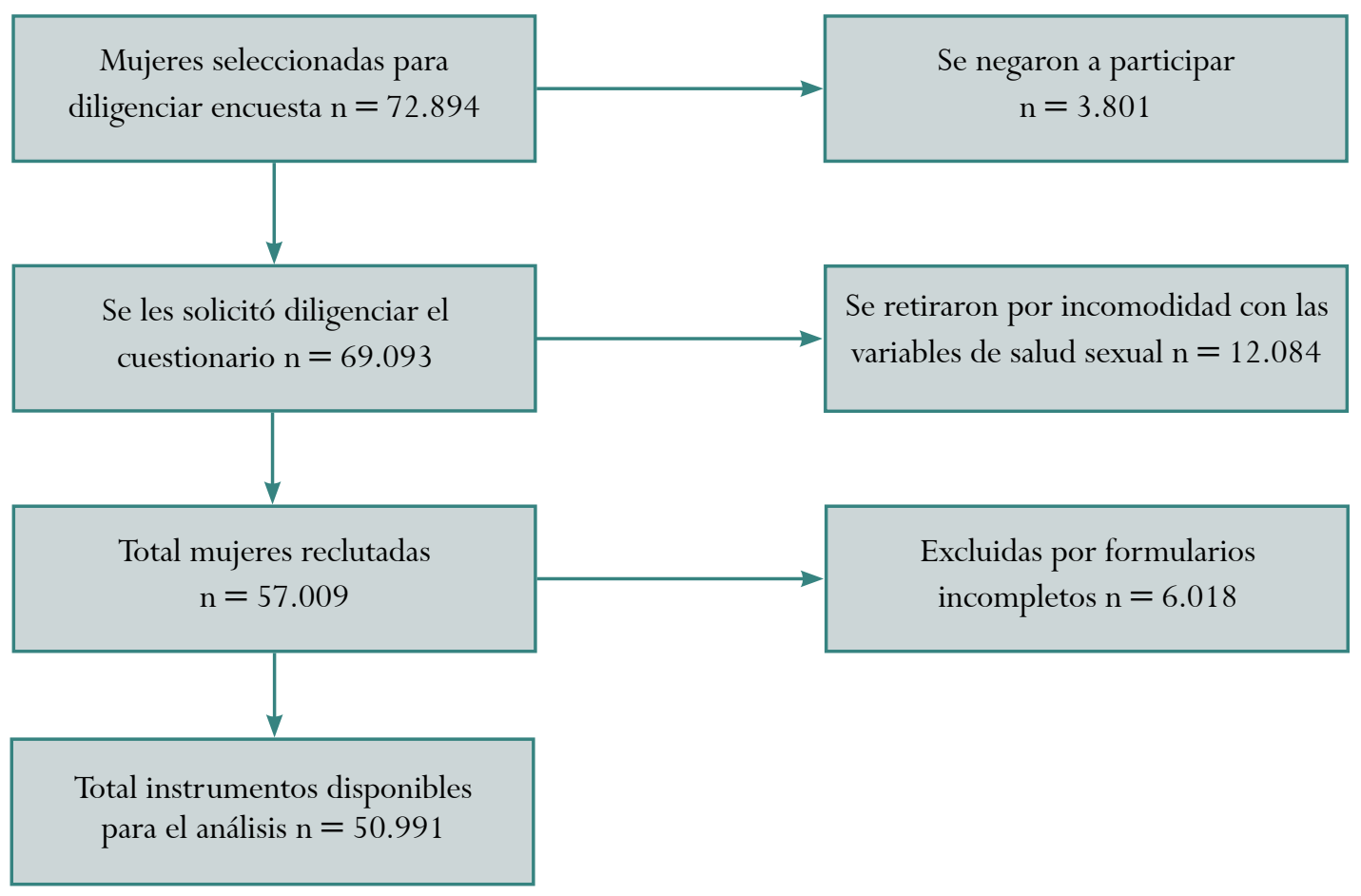


muestran en la tabla 1. La orientación sexual es predominantemente heterosexual (82,98\%). En relación con el inicio de la actividad sexual, la edad promedio es de 16,2 años (DE $\pm 2,1)$. La práctica sexual más frecuente es el coito vaginal, y la menos frecuente el coito anal (23,61\%); la masturbación es considerada una práctica común en el 77,34\% de las mujeres encuestadas. A la pregunta ¿cuántas veces tuvo relaciones sexuales la semana pasada? (periodo definido como el lapso de los siete días anteriores), el 57,39\% del total de la población encuestada informó tener relaciones tres veces por semana; de estas, el 40,89\% lo hacen una vez por encuentro, el 28,56\% lo hacen dos veces, el 20,79\% lo hacen 3 veces, y 9,18\% lo hace 4 o más veces por encuentro.

El 8,56\% de las mujeres informó haber sufrido alguna forma de violencia sexual a lo largo de la vida, mientras que el 5,19\% reportó abuso sexual por parte de la pareja.

La puntuación del IFSF en la población global del total de las 50.991 mujeres fue de 28,13 puntos; siendo el puntaje mayor de 34,26 y el mínimo de 7,83 puntos, con una desviación estándar de $\pm 6,12$ puntos. La prevalencia de disfunción sexual en el grupo estudiado fue del 32,97\% ( $\mathrm{n}=16.812 / 50.991)$, con una puntuación del IFSF de 24,07 $\pm 6,18$ puntos. La disfunción sexual presente con más frecuencia fue el trastorno del deseo, con 16.812 casos (32,97\%), seguido por la alteración del orgasmo con 11.178 casos $(21,93 \%)$ y, en tercer lugar, la alteración de la excitación con 8.562 casos (16,79\%). Se encontró que el 8,68\% presenta una disfunción sexual, el 64,16\% dos y 24,17\% 3 o más disfunciones sexuales, con una mediana de disfunciones sexuales por mujer de 2 (rango entre 1 y 4). En la tabla 2 se describe la puntuación detallada de cada dominio del IFSF en la población de mujeres con disfunción sexual. En cuanto a las alteraciones del orgasmo, el 19,67\% de las mujeres manifestó que nunca lo había experimentado en su vida; sin embargo, se observa una frecuencia de simuladoras del orgasmo del 58,34\%, contra un 31,14\% que "nunca" lo fingen, mientras que el 10,52 \% manifestó no saber qué es un orgasmo. A la pregunta acerca de la frecuencia para alcanzar el orgasmo, el 47,19\% dijo que "frecuentemente" llegaban al orgasmo, un 24,58\% que lo lograban "siempre" y el 6,98\% refirió ser multiorgásmica. El 4,89\% de las mujeres refirió haber asistido a una consulta de sexología o consejería sexual; de estas, el 2,85\% afirmó haber recibido, al menos una vez, tratamiento para el trastorno sexual.

$\mathrm{Al}$ analizar la población de mujeres con disfunción sexual, en menores y mayores de 40 años, se observa una prevalencia global de disfunciones sexuales del 21,08\% entre las menores de 40 años, y del 79,41 \% en las mayores de 40 años. Las mujeres menores de 40 años mostraron una prevalencia de 23,7 \% de trastornos del deseo, 14,1\% dificultades con la excitación, 11,4\% falla en la lubricación, 19,8\% dificultades con el orgasmo, 87,6\% satisfacción sexual y 5,4\% dolor con el coito; con una mediana de 2 disfunciones sexuales por mujer. En las mujeres mayores de 40 años la prevalencia observada fue de 38,4\% de trastornos del deseo, 16,2 \% dificultades con la excitación, 17,7 \% falla en la lubricación, 23,1\% dificultades con el orgasmo, $79,2 \%$ satisfacción sexual y $11,7 \%$ dolor con el coito, con una mediana de 3 disfunciones sexuales por mujer. En cuanto al puntaje por dominio se encuentran menores valores en las mujeres mayores de 40 años, tanto en los dominios como en el puntaje final del IFSF $(24,15 \pm 6,27$ vs. 23,64 \pm 5,49, respectivamente), como en los puntajes de cada dominio (tabla 3).

\section{DISCUSIÓN}

En este estudio se encontró una prevalencia del $32,97 \%$ de disfunciones sexuales en las mujeres colombianas. Se encuentra además que la disfunción sexual con más prevalencia es el trastorno del deseo $(32,97 \%)$, seguido por la alteración del orgasmo (21,93 \%) y la alteración de la excitación (16,79\%). Asimismo, se observó que es inusual la presencia de una sola disfunción sexual (8,69\%). 
Tabla 1.

Características sociodemográficas y en salud sexual y reproductiva de la mujeres encuestadas en función sexual en 12 ciudades en Colombia, 2009-2016

\section{Variables}

Edad* $^{*}$

Edad de la pareja*

Peso*

Talla*

IMC ${ }^{*}$

\section{Raza}

Mestizas

Afrocolombianas

Indígenas

Estrato socioeconómico

Alto

Medio

Bajo

\section{Estado civil}

Casada

Unión libre

Soltera

Divorciada

\section{Ocupación}

Amas de casa

Empleadas

Pensionadas

\section{Afiliación a seguridad social}

Contributivo

Subsidiado

Antecedente personal de depresión

Antecedente familiar de depresión

Antecedente de disfunción sexual

\section{Origen}

Urbano

Rural

\section{Nivel de estudio}

Secundaria

Técnica

Profesionales

\section{Orientación sexual}

Heterosexual

Homosexual

Bisexual

"Media \pm desviación estándar
$\mathbf{N}=50.991$

$$
30,8 \pm 18,9
$$

$45,6 \pm 5,4$

$60,3 \pm 10,8$

$1,59 \pm 0,51$

$23,7 \pm 4,2$

n (\%)

$30237(59,29 \%)$

12237 (\%)

$8517(16,7)$

$$
\begin{gathered}
8235(16,14 \%) \\
30978(60,75 \%) \\
11778(\%)
\end{gathered}
$$

$24795(48,62 \%)$

$14441(28,32)$

$2608(5,11 \%)$

$9147(17,93 \%)$

$26832(52,62 \%)$

$16465(32,29 \%)$

7694 (15,08\%)

$36861(72,28 \%)$

$14130(27,71 \%)$

$7164(14,04 \%)$

$6138(12,03 \%)$

$3708(7,27 \%)$

$40371(79,17 \%)$

$10620(20,82 \%)$

$27558(54,04 \%)$

$5538(10,86 \%)$

$17895(35,09 \%)$

$42237(82,83 \%)$

3057 (5,99\%)

(11,17\%) 


\begin{tabular}{|l|c|c|}
\hline \multicolumn{2}{|c|}{$\begin{array}{c}\text { Tabla } 2 . \\
\text { Índice de función sexual femenina en mujeres con disfunciones sexuales en } 12 \text { ciudades } \\
\text { en Colombia, 2009- 2016 }\end{array}$} \\
\hline \multicolumn{1}{|c|}{ Dominios } & Media ( \pm DE) & Disfunción sexual (\%) \\
\hline Deseo & $3,42 \pm 1,11$ & 32,97 \\
\hline Excitación & $4,08 \pm 0,87$ & 16,86 \\
\hline Lubricación & $4,26 \pm 0,93$ & 14,79 \\
\hline Orgasmo & $3,51 \pm 1,26$ & 21,93 \\
\hline Satisfacción & $4,41 \pm 0,84$ & 82,26 \\
\hline Dolor & $4,38 \pm 1,17$ & 7,56 \\
\hline Total puntaje & $24,07 \pm 6,18$ puntos & \\
\hline
\end{tabular}

Al comparar la prevalencia de disfunciones sexuales en este estudio se encuentran cifras similares a otras investigaciones realizadas en Colombia por Espitia en el Eje Cafetero, con un 34,9\% (12), y Monterrosa et al. con el 38,4\% (16). Al comparar nuestros resultados con otros autores en Latinoamérica, que también utilizaron el IFSF, se encuentra que los nuestros son inferiores a los reportados por Matute et al. en Ecuador con el 60,4\% (25), Abdo et al. en Brasil con el $49 \%$ (26), Castelo-Branco et al. en Chile con el 50,6\% (27), García et al. en Colombia con un 55,8\% (28). La diferencia entre los resultados obtenidos en nuestra investigación y los otros autores puede deberse al tipo de población seleccionada (25, 26), a la desigualdad de los grupos etarios (27), y a diferencias en aspectos raciales (14).

En relación con los dominios de IFSF, el deseo sexual se destacó como la disfunción más comúnmente reportada, resultado similar a otras publicaciones de diferentes países (14, 29-32). El alto porcentaje de anorgasmia en este grupo de mujeres (21\%) es inferior a lo publicado en la literatura colombiana $(22,23,33,34)$, excepto un estudio realizado en Bogotá en el año 2008, donde Acuña et al. encontraron un 3,29\% de mujeres con anorgasmia primaria (35); mientras que los trastornos del orgasmo, en la población de mujeres mayores de 40 años, son superiores a los obtenidos en otras investigaciones $(22,23)$. En cuanto a estudios publicados en otros países, nuestros resultados son similares a los encontrados en Brasil (21\%) (26) y a los informados por Najafabady et al., en Irán (26,1\%) (36). Sin embargo, muy inferiores a lo reportado por Ojomu et al. en Nigeria, quienes señalaron que el $55 \%$ de las mujeres tenía problemas con el orgasmo (37). Ellos consideran que la alta frecuencia podría estar asociada a la pobre comunicación conyugal, falta de juegos preliminares y a la religión islámica de este país. Este estudio orienta sobre la importancia de los actos religiosos o culturales en cuanto a la función sexual.

La prevalencia de los trastornos de la excitación sexual y la lubricación oscila en porcentajes similares a los publicados por otros autores $(13,38)$. El hallazgo de dolor coital (7,5\%) en nuestro estudio coincide con publicaciones internacionales de países desarrollados $(15,39,40)$.

Las altas puntuaciones del dominio satisfacción se relacionaron con la consistencia orgásmica, así como con la mayor frecuencia de relaciones sexuales. Esta última está sujeta a la influencia de múltiples aspectos del funcionamiento sexual femenino, lo cual es congruente con lo documentado en otros estudios (12, 13, 41-43). 


\begin{tabular}{|c|c|c|c|c|c|c|c|c|}
\multicolumn{7}{|c|}{ Tabla 3. } \\
\multicolumn{7}{|c|}{ Indice de Función Sexual Femenina (FSFI), por edades, en 12 ciudades en Colombia, $2009-2016$} \\
\hline & Deseo & Excitación & Lubricación & Orgasmo & Satisfacción & Dolor & Total \\
\hline $\begin{array}{c}<40 \\
\text { años }\end{array}$ & $3,36 \pm 0,81$ & $4,32 \pm 1,05$ & $4,17 \pm 1,17$ & $3,48 \pm 1,08$ & $4,71 \pm 1,14$ & $4,11 \pm 1,02$ & $24,15 \pm 6,27$ \\
\hline $\begin{array}{l}>40 \\
\text { años }\end{array}$ & $3,15 \pm 0,78$ & $4,05 \pm 0,75$ & $4,02 \pm 0,81$ & $3,39 \pm 0,93$ & $4,32 \pm 1,05$ & $4,71 \pm 1,08$ & $23,64 \pm 5,49$ \\
\hline
\end{tabular}

Respecto a nuestros hallazgos, existe una mayor prevalencia de disfunciones sexuales $(79,41 \%)$ en las mujeres mayores de 40 años frente a las menores de 40 años $(21,08 \%)$, con predominio de los trastornos del deseo en ambos grupos: 38,4 y 23,7\%, respectivamente. Se han reportado prevalencias similares de trastornos del deseo sexual en mujeres colombianas menores de 40 años, desde el 24,7\% (22) hasta el $75 \%$ en las mayores de 40 años $(14,23)$. Por otra parte, se observó que la frecuencia de la actividad sexual se vio disminuida con el aumento de la edad, siendo mayor entre las mujeres de menos de 40 años y menor en las de más de 40 años, tal como lo describe la literatura revisada $(12,44,45)$.

Respecto a las limitaciones de esta investigación se encontró que el cuestionario utilizado amerita cierto nivel educativo que le facilite a la mujer una adecuada comprensión e interpretación de las preguntas y los dominios, dado que no es apropiado para uso en población analfabeta o con bajo nivel de escolaridad; por tanto, al no haberse incluido esta población, se pudo incurrir en un sesgo de selección, detalle que podría generar un sesgo en la alta participación de mujeres con escolaridad elevada, descrita en la población del estudio.

El haber hecho un muestreo por conveniencia limita la generalización de los resultados; asimismo, al no haber extendido el estudio al resto de las ciudades del país se podría estar excluyendo a muchas más mujeres con disfunciones sexuales, situación que podría introducir un sesgo en la prevalencia a nivel de las mujeres colombianas. No obstante, debido a que la muestra fue representativa, se tiene información sobre un importante grupo poblacional.
Próximos estudios sobre la prevalencia de disfunciones sexuales en mujeres colombianas deberían realizarse mediante muestreos aleatorios en población general, ya que seleccionar sujetos en consulta de ginecología podría llevar a sesgos de selección. Sería importante tener mayor participación de las ciudades del país, y contar con participación de población rural.

Como fortalezas de este estudio se consideran que la población es una muestra grande, la cual fue seleccionada de mujeres que asistían a consulta ginecológica, con una holgada variedad de edades y estratos socioeconómicos, así como contar con la utilización del IFSF, un reconocido cuestionario de comprobada confiabilidad y consistencia interna, validado recientemente en Colombia (23).

A la luz de estos resultados invitamos a los médicos a que rutinariamente pregunten sobre los posibles trastornos sexuales de sus pacientes, ya que el $50 \%$ de las mujeres que son interrogadas suelen expresar problemas sexuales (46). Al respecto, los ginecólogos se encuentran en una posición privilegiada, ya que entre el $42 \%$ (47) y 98,8\% (48) de las mujeres discuten sus preocupaciones sexuales con sus ginecólogos durante los chequeos de rutina; por tanto, estos pueden abordar dichas preocupaciones con mayor facilidad, y pueden ayudarlas a resolver sus dificultades sexuales.

\section{CONCLUSIÓN}

En las mujeres colombianas existe una prevalencia de disfunciones sexuales cercana a la tercera parte de la población, caracterizadas principalmente por trastornos del deseo y del orgasmo, lo que repercute 
negativamente en la calidad de vida. Es importante indagar por aspectos de la sexualidad en la consulta de ginecología para establecer un plan de manejo interdisciplinario.

\section{AGRADECIMIENTOS}

Agradezco a las mujeres participantes en esta investigación, a las instituciones que facilitaron sus instalaciones, al personal de enfermería, a las trabajadoras sociales, psicólogas, bioestadísticos y epidemiólogos que nos apoyaron. Asimismo, reconozco la labor de mi maestro y amigo, el doctor Guillermo Sánchez Barea, por contagiarme con su motivación de sacar adelante la sexología en Colombia.

\section{REFERENCIAS}

1. Organización Panamericana de la salud (OPS), Organización Mundial de la Salud (OMS). Promoción de la salud sexual: recomendaciones para la acción. Antigua Guatemala: OPS, OMS; 2000.

2. Cavalcanti IF, Farias PD, Ithamar L, Silva VM, Lemos A. Sexual function and factors associated with sexual dysfunction in climacteric women. Rev Bras Ginecol Obstet. 2014;36:497-502. https://doi.org/10.1590/ S0100-720320140004985.

3. Kammerer-Doak D, Rogers RG. Female sexual function and dysfunction. Obstet Gyn Clin N Am. 2008;35:169-83. https://doi.org/10.1016/j. ogc.2008.03.006

4. Dunn KM, Croft PR, Hackett GI. Association of sexual problems with social, psychological, and physical problems in men and women: A cross sectional population survey. J Epidemiol Community Health. 1999;53: 144-8. https://doi.org/10.1136/jech.53.3.144

5. Hedelin H, Abramsson L. [Sexuality-an important factor for quality of life. Who should treat erectile dysfunction?]. Lakartidningen. 1997;94:2548-52.

6. Lammerink EAG, de Bock GH, Pascal A, van Beek AP, van den Bergh ACM, Sattler MGA, et al. A survey of female sexual functioning in the general dutch population. J Sex Med. 2017;14:937-49. https://doi. org/10.1016/j.jsxm.2017.04.676
7. Nappi RE, Nijland EA. Women's perception of sexuality around the menopause: Outcomes of a European telephone survey. Eur J Obstet Gynecol Reprod Biol. 2008;137:10-6. https://doi.org/10.1016/j. ejogrb.2006.10.036

8. Shin H, Min B, Park J, Son H. A 10-year interval study to compare the prevalence and risk factors of female sexual dysfunction in Korea: The Korean internet sexuality survey (KISS) 2014. Int J Impot Res. 2017;29:49-53. https://doi.org/10.1038/ijir.2016.41.

9. Singh JC, Tharyan P, Kekre NS, Singh G, Gopalakrishnan G. Prevalence and risk factors for female sexual dysfunction in women attending a medical clinic in south India. J Postgrad Med. 2009;55:113-20. https:// doi.org/10.4103/0022-3859.52842.

10. Valadares AL, Lui-Filho JF, Costa-Paiva L, PintoNeto AM. Middle-aged female sexual dysfunction and multimorbidity: A population-based study. Menopause. 2016;23:304-10. https://doi.org/10.1097/ GME.0000000000000533

11. Chedraui P, Pérez-López FR, Sánchez H, Aguirre W, Martínez N, Miranda O. Assessment of sexual function of mid-aged ecuadorian women with the 6-item Female Sexual Function Index. Maturitas. 2012;71:40712. https://doi.org/10.1016/j.maturitas.2012.01.013

12. Shifren JL, Monz BU, Russo PA, Segreti A, Johannes CB. Sexual problems and distress in United States women: Prevalence and correlates. Obstet Gynecol. 2008;112:970-8. https://doi.org/10.1097/ AOG.0b013e3181898cdb

13. Laumann EO, Paik A, Rosen RC. Sexual dysfunction in the United States: Prevalence and predictors. JAMA. 1999;281:537-44. https://doi.org/10.1001/ jama.281.6.537

14. Monterrosa-Castro Á, Márquez-Vega J, Arteta-Acosta C. Disfunción sexual en mujeres climatéricas afrodescendientes del Caribe Colombiano. IATREIA. 2014; 27:31-41.

15. Dennerstein L, Hayes RD. Confronting the challenges: Epidemiological study of female sexual dysfunction and the menopause. J Sex Med 2005;2(Suppl 3):118-32. https://doi.org/10.1111/j.1743-6109.2005.00128.x 
16. Lewis RW, Fugl-Meyer KS, Bosch R, et al. Epidemiology/Risk factors of sexual dysfunction. J Sex Med. 2004;1:35-9. https://doi.org/10.1111/j.17436109.2004.10106.x

17. Simons JS, Carey MP. Prevalence of sexual dysfunctions: Results from a decade of research. Arch Sex Behav. 2001;30:177-219. https://doi. org/10.1023/A:1002729318254

18. Quirk FH, Heiman JR, Rosen RC, Laan E, Smith MD, Boolell M. Development of a sexual function questionnaire for clinical trials of female sexual dysfunction. J Womens Health Gend Based Med. 2002;11:277-89. https://doi.org/10.1089/152460902753668475

19. Taylor JF, Rosen RC, Leiblum SR. Self-report assessment of female sexual function: Psychometric evaluation of the Brief Index of Sexual Functioning for Women. 1994;23:627-43.

20. Rosen R, Brown C, Heiman J, Leiblum S, Meston C, Shabsigh R, et al. The Female Sexual Function Index (FSFI): A multidimensional selfreport instrument for the assessment of female sexual function. J Sex Marital Ther. 2000;26:191-208. https://doi. org/10.1080/009262300278597.

21. Vallejo-Medina P, Pérez-Durán C, Saavedra-Roa A. Translation, adaptation, and preliminary validation of the female sexual function index into spanish (Colombia). Arch Sex Behav. 2017;1-14. https://doi. org/10.1007/s10508-017-0976-7

22. Espitia De La Hoz FJ. Prevalencia de disfunción sexual en mujeres del Eje Cafetero. Rev Cienc Biomed. 2016;7:25-33

23. Espitia De La Hoz FJ. Evaluación de la prevalencia de disfunción sexual en mujeres médicos del Eje Cafetero colombiano, en etapa de climaterio. Archivos de Medicina (Col). 2017;17:70-7.

24. Hendrickx L, Gijs L, Enzlin P. Sexual difficulties and associated sexual distress in Flanders (Belgium): A representative population-based survey study. J Sex Med. 2016;13:650-68. https://doi.org/10.1016/j. jsxm.2016.01.014

25. Matute V, Arévalo C, Espinoza A. Estudio transversal: prevalencia de disfunción sexual femenina y factores asociados en pacientes del hospital "José Carrasco
Arteaga". Rev Med HJCA. 2016;8:19-24. https:/doi. org/10.14410/2016.8.1.ao.03

26. Abdo CH, Oliveira WM, Jr, Moreira ED, Jr, Fittipaldi JA. Prevalence of sexual dysfunctions and correlated conditions in a sample of Brazilian women-results of the Brazilian study on sexual behavior (BSSB). Int J Impot Res. 2004;16:160-6. https://doi.org/10.1038/ sj.ijir.3901198

27. Castelo-Branco C, Blümel JE, Araya H, Riquelme R, Castro G, Haya J, et al. Prevalence of sexual dysfunction in a cohort of middle-aged women: Influences of menopause and hormone replacement therapy. J. Obstet Gynaecol. 2003;23:426-30. https://doi. org/10.1080/0144361031000120978

28. García, SP, Aponte HA, Socorro Moreno PS. Diagnóstico de la disfunción sexual femenina y su correlación con el perfil hormonal en la población femenina que consulta a los servicios de urología, ginecología y personal femenino del hospital de San José, en Bogotá, Colombia. Urol Colomb. 2005;14:75-80.

29. Sheehan DV, Lecrubier Y, Sheehan KH, Amorim P, Janavs J, Weiller E, et al. The Mini-International Neuropsychiatric Interview (M.I.N.I.): the development and validation of a structured diagnostic psychiatric interview for DSM-IV and ICD-10. J Clin Psychiatry. 1998;59:34-57. https://doi.org/10.1016/S0924-9338(97)83296-8

30. Hayes RD, Bennett CM, Fairley CK, Dennerstein L. What can prevalence studies tell us about female sexual difficulty and dysfunction? J Sex Med. 2006;3:589-95. https://doi.org/10.1111/j.1743-6109.2006.00241.x

31. Oksuz E, Malhan S. Prevalence and risk factors for female sexual dysfunction in Turkish women. J Urol. 2006;175:654-58: discussion 8.7. https://doi. org/10.1016/S0022-5347(05)00149-7

32. Basson R. Clinical practice. Sexual desire and arousal disorders in women. N Engl J Med. 2006;354:1497506. https://doi.org/10.1056/NEJMcp050154

33. Guarín-Serrano R, Cadena-Afanador L, Mujica-Rodríguez A, Ochoa-Vera M, Useche-Aldana B. Prevalencia de orgasmo en mujeres universitarias de Bucaramanga (Colombia), 2013. Rev Colomb Obstet Ginecol. 2014;65:330-37. https://doi.org/10.18597/rcog.37 
34. Quintero MT, Gómez M, Uribe JF. Perfil orgásmico en universitarias de ciencias de la salud. Urol Colomb. 2013;22:18-29.

35. Acuña A, Ceballos MP, Suárez PA. Estudio sobre algunos aspectos del comportamiento sexual femenino. Urol Colomb. 2008;17:79-90.

36. Najafabady MT, Salmani Z, Abedi P. Prevalence and related factors for anorgasmia among reproductive aged women in Hesarak, Iran. Clinics. 2011;66:83-6. https:// doi.org/10.1590/S1807-59322011000100015

37. Ojomu F, Thacher T, Obadofin M. Sexual problems among married Nigerian women. International Journal of Impotence Research. 2007;19:310-6. https:// doi.org/10.1038/sj.ijir.3901524

38. Moynihan R. The making of a disease: Female sexual dysfunction. BMJ. 2003;326:45-7. https://doi. org/10.1136/bmj.326.7379.45

39. Danielsson I, Sjoberg I, Stenlund H, Wikman M. Prevalence and incidence of prolonged and severe dyspareunia in women: Results from a population study. Scand J Public Health. 2003;31:113-8. https:// doi.org/10.1080/14034940210134040

40. Schultz W, Basson R, Binik Y, Eschenbach D, Wesselmann U, van Lankveld J. Women's sexual pain and its management. J Sex Med. 2005;2:301-16. https:// doi.org/10.1111/j.1743-6109.2005.20347.x

41. Hurlbert DF, Apt C, Rabehl SM. Key variables to understanding female sexual satisfaction: An examination of women in nondistressed marriages. J Sex Marital Ther. 1993;19:154-65. https://doi. org/10.1080/00926239308404899
42. Burri A, Spector T. Recent and lifelong sexual dysfunction in a female UK population sample: Prevalence and risk factors. J Sex Med. 2011;8:2420-30. https://doi. org/10.1111/j.1743-6109.2011.02341.x

43. Figueroa R, Jara D, Fuenzalida A, del Prado M, Flores D, Blumel J. Prevalencia de disfunción sexual en mujeres climatéricas. Rev Méd Chile. 2009;137:345-50. https:// doi.org/10.4067/S0034-98872009000300004.

44. Shams Nateri N, Ashraf Kazemi MB, Shirinkam F. Women coping strategies towards menopause and its relationship with sexual dysfunction. Iran J Nurs Midwifery Res. 2017;22:343-7. doi: 10.4103/ijnmr. IJNMR_234_15.

45. Rosen RC, Connor MK, Miyasato G, et al. Sexual desire problems in women seeking healthcare: A novel study design for ascertaining prevalence of hypoactive sexual desire disorder in clinic-based samples of U.S. women. J Womens Health (Larchmt). 2012;21:50515. https://doi.org/10.1089/jwh.2011.3002

46. Collier F, Cour F. How to manage a woman with a sexual complaint in clinical practice? Prog Urol. 2013;3:612-20. https://doi.org/10.1016/j.purol. 2012.09.018

47. Berman L, Berman J, Felder S, et al. Seeking help for sexual function complaints: What gynecologists need to know about the female patient's experience. Fertil Steril. 2003;79:572-6. https://doi.org/10.1016/ S0015-0282(02)04695-2

48. Nussbaum MR, Gamble G, Skinner B, Heiman J. The high prevalence of sexual concerns among women seeking routine gynecological care. J Fam Pract. 2000;49:229-32. 


\begin{tabular}{|c|c|c|c|c|c|}
\hline \multicolumn{6}{|c|}{$\begin{array}{c}\text { Anexo } 1 . \\
\text { Índice de Función Sexual Femenina (IFSF) }\end{array}$} \\
\hline Dominio & Preguntas & Puntaje & Factor & Mínimo & Máximo \\
\hline Deseo & $1-2$ & $1-5$ & 0,6 & 1,2 & 6 \\
\hline Excitación & $3-6$ & $0-5$ & 0,3 & 0 & 6 \\
\hline Lubricación & $7-10$ & $0-5$ & 0,3 & 0 & 6 \\
\hline Orgasmo & $11-13$ & $0-5$ & 0,4 & 0 & 6 \\
\hline Satisfacción & $14-16$ & $0-5$ & 0,4 & 0,8 & 6 \\
\hline Dolor & $17-19$ & $0-5$ & 0,4 & 0 & 6 \\
\hline \multicolumn{4}{|c|}{ Puntaje total } & 2 & 36 \\
\hline
\end{tabular}

